\title{
A case of thoracic endovascular aortic repair for acute retrograde type A aortic dissection with paraplegia
}

Satoshi Nishi, MD, Daisuke Arima, MD, Akihiro Yoshimoto, MD, and Yoshihiro Suematsu, MD, PhD

From the Department of Cardiovascular Surgery, Tsukuba Memorial Hospital, Tsukuba, Japan.

Disclosures: The authors reported no conflicts of interest.

The Journal policy requires editors and reviewers to disclose conflicts of interest and to decline handling or reviewing manuscripts for which they may have a conflict of interest. The editors and reviewers of this article have no conflicts of interest.

Received for publication Feb 4, 2020; revisions received Feb 4, 2020; accepted for publication March 19, 2020; available ahead of print April 2, 2020.

Address for reprints: Satoshi Nishi, MD, Tsukuba Memorial Hospital, 1187-299, Kaname, Tsukuba-shi, Ibarakiken, Japan (E-mail: stscnm0808@yahoo.co.jp).

JTCVS Techniques 2020;2:20-2

2666-2507

Copyright $\Subset 2020$ The Authors. Published by Elsevier Inc. on behalf of The American Association for Thoracic Surgery. This is an open access article under the CC BY-NC-ND license (http://creativecommons.org/licenses/bync-nd/4.0/).

https://doi.org/10.1016/j.xjtc.2020.03.016

Despite a recent increasing trend in the number of reports of thoracic endovascular aortic repair (TEVAR) for acute retrograde type $A$ aortic dissection (ARAAD), TEVAR for ARAAD with paraplegia has not been reported yet. ${ }^{1}$ We herein report a case of TEVAR for ARAAD with paraplegia.

\section{CASE REPORT}

A 48-year-old man was referred to our hospital with a diagnosis of acute type A aortic dissection with paraplegia. The paraplegia was identified 3 hours before the patient arrived at our hospital. The degree of paraplegia of the right and left lower extremities were manual muscle testing grade 2 and 3, respectively. The patient had a history of operation for atrial septal defect and did not have Marfan syndrome. A preoperative echocardiogram and an intraoperative transesophageal echocardiogram were obtained, which showed no evidence of aortic regurgitation or pericardial effusion. Contrast-enhanced thin-slice computed tomography showed aortic dissection from the ascending aorta (AAo) to the right external iliac artery (rEIA) without other visceral ischemia. The AAo was $45 \mathrm{~mm}$ in diameter, including a thrombosed false lumen (FL) $7 \mathrm{~mm}$ in thickness (Figure 1,A). The FL of the AAo and aortic arch was thrombosed, and that from the descending aorta (DAo) to the rEIA was patent. Primary entry tear (pET) was located in the DAo at T8 (Figure $1, B$ ), and other entry tears were located in the DAo at T12, abdominal aorta, and the rEIA.

As the risk of rupture of the AAo was considered to be low, we planned to perform TEVAR followed by spinal drainage. An intravascular ultrasound was performed in the operating room. The covered stents Zenith TX2 ZTEG-2P-30-120-PF and ESBE-24-80-T-PF (Cook, Bloomington, Ind) were deployed from the left subclavian artery to the T9 with a length of $170 \mathrm{~cm}$, whereas the

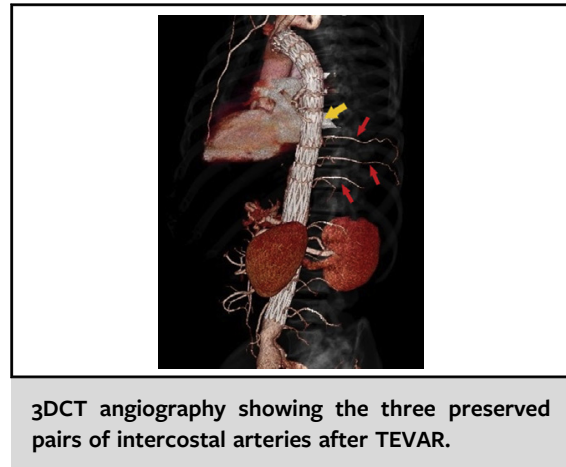

CENTRAL MESSAGE

TEVAR for acute retrograde type

A aortic dissection with visceral

malperfusion can be an effective

minimally invasive treatment in

select cases.

See Commentaries on pages 23 and 25 .

uncovered stents Zenith Dissection GZSD-36-123-2 and GZSD-36-164-2 (Cook) were deployed from the T9 to L4 with a length of $260 \mathrm{~cm}$ to close the pET, intentionally to preserve 3 pairs of patent intercostal arteries originating from the true lumen (TL) and increase the blood flow from the TL to the preserved or patent intercostal, lumbar, and hypogastric arteries. Spinal drainage was promptly performed after TEVAR in the operating room.

The patient's paraplegia gradually improved. He became able to stand up by himself and walk with a walking aid on postoperative day (POD) 3. The spinal drain was removed on POD 5. Contrast-enhanced computed tomography on POD 8 showed the AAo and DAo with an expanded TL and reduced FL as well as preservation of the 3 pairs of intercostal arteries (Figure 2, $A$ and $B$ ). He was discharged ambulatory on POD 20. His bladder and rectal disturbance had completely recovered by 9 months and 3 weeks later, respectively. He remained an outpatient without adverse aortic events and showed remodeling from the AAo to the proximal DAo 1 year after TEVAR. The patient provided informed consent for the publication of the study data. 

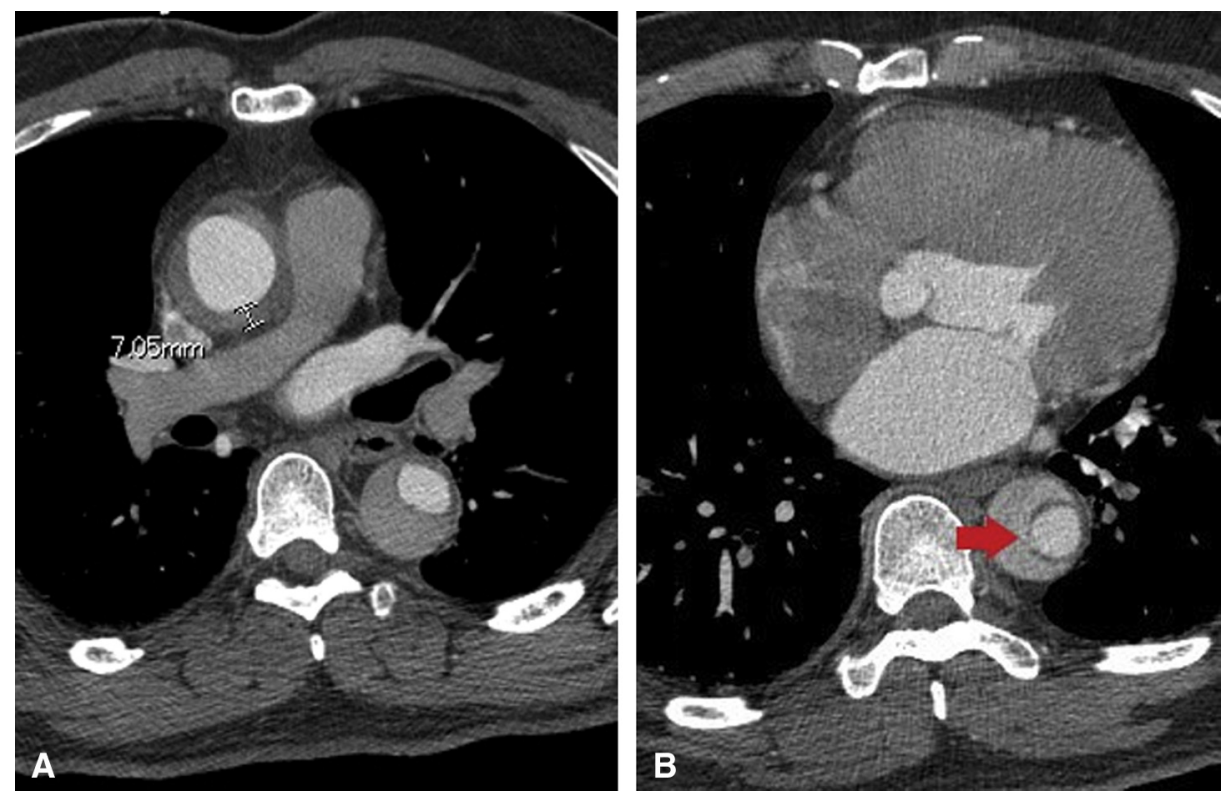

FIGURE 1. A, Preoperative contrast-enhanced thin-slice computed tomography showed dissection of the ascending aorta $45 \mathrm{~mm}$ in size, including a thrombosed false lumen $7 \mathrm{~mm}$ in thickness, the descending aorta with a narrowed true lumen and partially thrombosed false lumen, and the lack of an entry tear located in the ascending aorta. B, Preoperative contrast-enhanced thin-slice computed tomography showed the primary entry tear (red arrow) located in the descending aorta at the $\mathrm{T} 8$ level where the false lumen was more strongly enhanced by contrast medium than that of the proximal descending aorta.

\section{DISCUSSION}

This strategy was prepared for patients with organ or limb ischemia who had ARAAD with an AAo diameter $<50 \mathrm{~mm}$ and thrombosed FL $<10 \mathrm{~mm}$ in thickness. ${ }^{2}$ In the present case, a cardiopulmonary bypass circuit and equipment were prepared because we originally planned to perform AAo replacement. However, we revised our plan based on our criteria, as the most important issue with this young
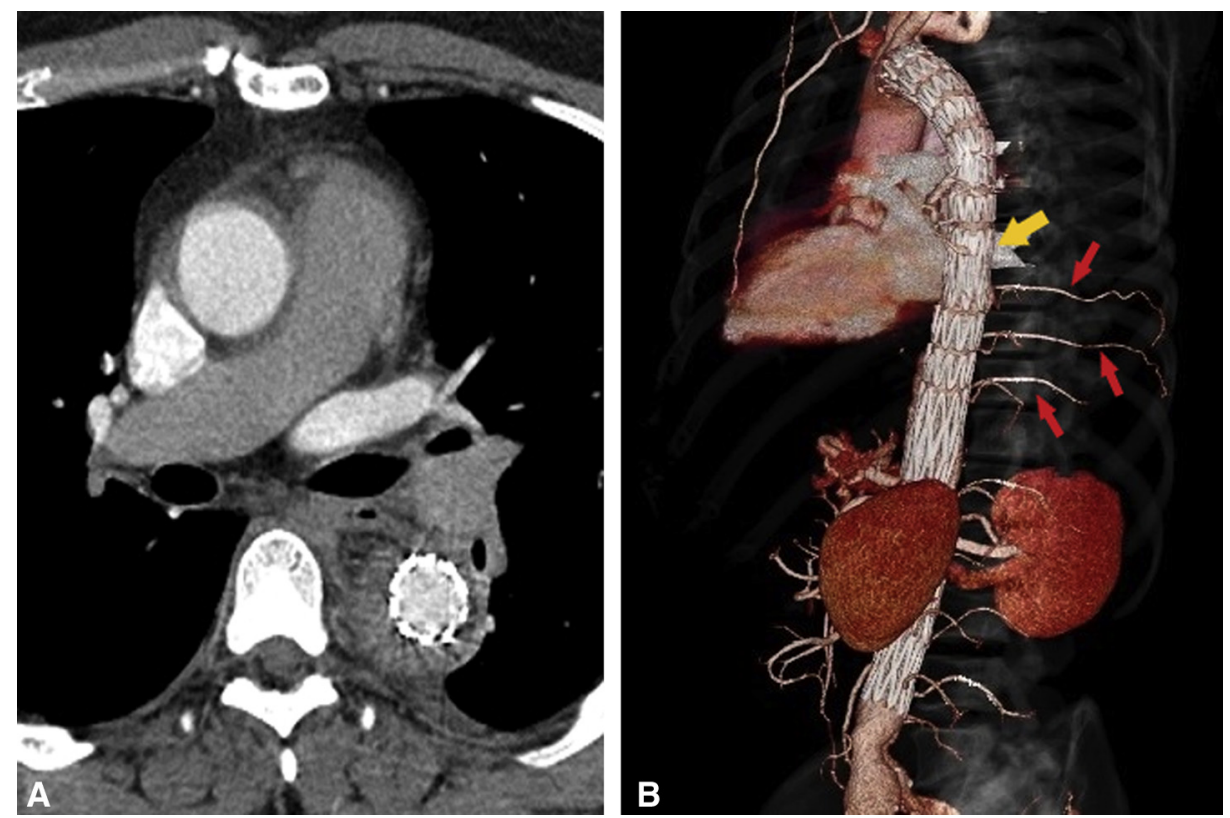

FIGURE 2. A, Contrast-enhanced computed tomography on postoperative day 8 showed the ascending and descending aorta with an expanded true lumen and reduced false lumen. B, Three-dimensional computed tomography angiography on postoperative day 8 showed that the covered stents were deployed from the left subclavian artery to the T9 level with closure of the primary entry tear located in the descending aorta at the T8 level (yellow arrow), and the uncovered stents were deployed from the T9 to L4 level with preservation of the 3 pairs of patent intercostal arteries originating from the true lumen (red arrows). 
patient who did not have Marfan syndrome was improving the paraplegia. We decided against open or hybrid surgery including the frozen elephant trunk (FET) technique because the pET was located at the T8 level, which would have required the use of a longer FET, potentially increasing the risk of spinal cord ischemia. ${ }^{3}$ Tsagakis and colleagues ${ }^{4}$ performed endovascular true lumen stabilization before surgery using self-expandable uncovered stents to restore abdominal organ perfusion for acute type I aortic dissection with malperfusion. Although we considered performing staged open surgery if necessary, it was ultimately not needed.

The survival rate of TEVAR for ARAAD with an entry tear in DAo without complications was $100 \%$ during a mean follow-up period of 22 months. ${ }^{1}$

Although a stent graft-induced new entry tear at the proximal site of the stent graft is a critical complication that must be prevented, if TEVAR is performed with a stent graft without a proximal bare stent, as in our case, the graft can be used as a FET when open surgery is required. Therefore, TEVAR for ARAAD with paraplegia that developed due to malperfusion is a minimally invasive treatment, feasible, and effective in select cases. However, careful follow-up is needed, as there is no consensus concerning TEVAR for ARAAD, and the long-term outcomes are unclear.

\section{References}

1. Chu C, Wang T, Li QM, Li M, Jiang XH, Luo MY, et al. Thoracic endovascular aortic repair for retrograde type A aortic dissection with an entry tear in the descending aorta. J Vasc Interv Radiol. 2012;23:453-60.

2. Kitai T, Kaji S, Yamamuro A, Tani T, Tamita K, Kinoshita M, et al. Clinical outcomes of medical therapy and timely operation in initially diagnosed type A aortic intramural hematoma a 20-year experience. Circulation. 2009;120(suppl 1): S292-8.

3. Katayama K, Uchida N, Katayama A, Takahashi S, Takasaki T, Kurosaki T, et al. Multiple factors predict the risk of spinal cord injury after frozen elephant trunk technique for extended thoracic aortic disease. Eur J Cardiothorac Surg. 2015; 47:616-20.

4. Tsagakis K, Jánosi RA, Frey UH, Schlosser T, Chiesa R, Rassaf T, et al. True lumen stabilization to overcome malperfusion in acute type I aortic dissection. Semin Thorac Surg. 2018;31:740-8. 